\title{
Operator-valued zeta functions and Fourier analysis
}

\author{
Carl M. Bender ${ }^{1}$ and Dorje C. Brody ${ }^{2}$ \\ ${ }^{1}$ Department of Physics, Washington University, St Louis, MO 63130, USA \\ ${ }^{2}$ Department of Mathematics, University of Surrey, Guildford GU2 7XH, UK, and \\ Department of Optical Physics and Modern Natural Science, \\ St Petersburg National Research University of Information Technologies, \\ Mechanics and Optics, St Petersburg 197101, Russia
}

The Riemann zeta function $\zeta(s)$ is defined as the infinite sum $\sum_{n=1}^{\infty} n^{-s}$, which converges when $\operatorname{Re} s>1$. The Riemann hypothesis asserts that the nontrivial zeros of $\zeta(s)$ lie on the line $\operatorname{Re} s=\frac{1}{2}$. Thus, to find these zeros it is necessary to perform an analytic continuation to a region of complex $s$ for which the defining sum does not converge. This analytic continuation is ordinarily performed by using a functional equation. In this paper it is argued that one can investigate some properties of the Riemann zeta function in the region $\operatorname{Re} s<1$ by allowing operator-valued zeta functions to act on test functions. As an illustration, it is shown that the locations of the trivial zeros can be determined purely from a Fourier series, without relying on an explicit analytic continuation of the functional equation satisfied by $\zeta(s)$.

1. In the so-called Hilbert-Pólya programme, one attempts to establish the Riemann hypothesis by (a) finding an operator (possibly a differential operator) along with a boundary condition such that the eigenvalues of the operator correspond to the nontrivial zeros of the zeta function, and (b) showing that the operator is self-adjoint. A $\mathcal{P} \mathcal{T}$-symmetric operator fulfilling the requirement (a) has recently been identified [1] and its eigenvalues were subsequently shown to be real [2]. However, these findings have not yet brought us closer to a proof of the Riemann hypothesis because the span of the eigenfunctions of the operator satisfying the boundary condition may not be fully contained within the rigged Hilbert space upon which the self-adjointness of the operator is established. To be specific, although a Hilbert space has been identified, one is automatically restricting the analysis to the critical line $\operatorname{Re} s=\frac{1}{2}$ of the zeta function $\zeta(s)$ so that little can be inferred about the zeros off the critical line, if there are any. This may explain why the Hilbert-Pólya programme has not yet led to a proof of the Riemann hypothesis.

With this in mind, we propose here an alternative way to investigate the properties of the Riemann zeta function by analysing operator-valued zeta functions; that is, we examine the behaviour of $\zeta(\hat{s})$, where $\hat{s}$ is an operator, such as the dilation operator. It is unclear whether this approach will lead to a deep understanding of the zeta function. However, it is shown here that one can use this approach to establish some properties of Riemann zeta function very easily. Specifically, we can calculate $\zeta(s)$ for some integer values of $s$ without performing an explicit analytic continuation.

In the Hilbert-Pólya programme one investigates operators whose eigenvalues correspond to the locations of the zeros of the zeta function but we propose here to investigate operators whose eigenvalues are the values of the zeta function itself. For example, in the HilbertPólya programme one might consider the properties of the operator $\frac{1}{2}\left(1-\mathrm{i} \hat{h}_{\mathrm{BK}}\right)$, where $\hat{h}_{\mathrm{BK}}=\hat{x} \hat{p}+\hat{p} \hat{x}$ denotes the Berry-Keating Hamiltonian [3], but here we consider the operator $\zeta\left(\frac{1}{2}\left(1-\mathrm{i} \hat{h}_{\mathrm{BK}}\right)\right)$. We investigate such an operator by letting it act on trigonometric functions. 
We will show that

$$
\zeta\left(\frac{1}{2}\left(1-\mathrm{i} \hat{h}_{\mathrm{BK}}\right)\right) \sin x=\frac{\sin x}{2(1-\cos x)}
$$

for $x \in(0, \pi]$, from which we can deduce that $\zeta(-n)=(-1)^{n} B_{n+1} /(n+1)$ for $n$ a positiveodd integer, where $\left\{B_{n}\right\}$ are the Bernoulli numbers. There are numerous similar relations, from which further properties of the zeta function can be inferred. Another example is

$$
\zeta\left(\frac{1}{2}\left(3-\mathrm{i} \hat{h}_{\mathrm{BK}}\right)\right) \sin x=\frac{\pi-x}{2}
$$

for $x \in[0, \pi]$, from which we can deduce that $\zeta(s)$ vanishes for negative-even integers $s$ (these are the trivial zeros) without analytically continuing the functional equation $\zeta(s)=$ $2^{s} \pi^{s-1} \sin (\pi s / 2) \Gamma(1-s) \zeta(1-s)$. From (2) we can also deduce that $\zeta(0)=-\frac{1}{2}$, and that $\zeta(s)$ has a pole at $s=1$. We remark that operators of the form $\zeta\left(\frac{1}{2} \pm \frac{1}{2} \mathrm{i} \hat{h}_{\mathrm{BK}}\right)$ were mentioned briefly in Ref. [3].

2. This paper is based in part on the following relations in Fourier analysis [4]:

$$
\sum_{n=1}^{\infty} \frac{\cos (n x)}{n^{2 m}}=\frac{(-1)^{m-1}(2 \pi)^{2 m}}{2(2 m) !} B_{2 m}\left(\frac{x}{2 \pi}\right)
$$

and

$$
\sum_{n=1}^{\infty} \frac{\sin (n x)}{n^{2 m-1}}=\frac{(-1)^{m}(2 \pi)^{2 m-1}}{2(2 m-1) !} B_{2 m-1}\left(\frac{x}{2 \pi}\right)
$$

for $m=1,2, \ldots$, where $B_{m}(x)$ denotes the Bernoulli polynomial of order $m$. Note that these equations are only valid for real $x \in[0,2 \pi]$; the Fourier series diverge for complex $x$. If we set $m=1$ in (4), we obtain $(\pi-x) / 2$, which is the right side of (2). Similar series were investigated by Clausen [5].

In this paper we reinterpret these results by using quantum-mechanical operator techniques. We show that it is possible to infer properties of $\zeta(s)$ by studying actions of the operator $\zeta(\hat{s})$ on functions when $\hat{s}$ is an operator. (Bernoulli polynomials play a major role in the theory of the Riemann zeta function, so it is not surprising that some properties of the zeta function on the real line can be inferred in the context of Fourier series.)

Series of the form (3) or (4) can be extended to cases for which $m$ is 0 or a negative integer. Such series can be summed by using Euler summation. For example, we have

$$
\sum_{n=1}^{\infty} \mathrm{e}^{\mathrm{i} n x}=\lim _{r \rightarrow 1^{-}} \sum_{n=1}^{\infty}\left(r \mathrm{e}^{\mathrm{i} x}\right)^{n}=\lim _{r \rightarrow 1^{-}} \frac{r \mathrm{e}^{\mathrm{i} x}}{1-r \mathrm{e}^{\mathrm{i} x}}=\frac{1}{\mathrm{e}^{-\mathrm{i} x}-1} .
$$

Taking the imaginary part, we deduce that

$$
\sum_{n=1}^{\infty} \sin (n x)=\frac{\sin x}{2(1-\cos x)}
$$

which is the right side of (1). This result can also be obtained by complex analysis; from the analytic continuation of the Lerch zeta function

$$
L(s, x)=\sum_{n=1}^{\infty} \frac{\mathrm{e}^{\mathrm{i} n x}}{n^{s}}=\frac{\Gamma(1-s)}{2 \pi \mathrm{i}} \int_{C} \frac{\mathrm{e}^{t} t^{s-1}}{1-\mathrm{e}^{t+\mathrm{i} x}} \mathrm{~d} t,
$$


Apostol deduced sums such as (6) [6]. Here, the integration path $C$ is a Hankel contour that encircles the negative- $t$ axis in the positive direction. Thus, we obtain (5) by setting $s=0$ in (7) and using the residue at $t=0$ to evaluate the integral.

3. Let us proceed to establish relations such as (2) above. For this purpose we require the notion of the dilation operator. The generator of the dilation is $\hat{x} \hat{p}$, where $\hat{p}=-\mathrm{i} \mathrm{d} / \mathrm{d} x$, so that for a smooth function $f(x)$ we have

$$
\mathrm{e}^{\mathrm{i} \lambda \hat{x} \hat{p}} f(x)=f\left(\mathrm{e}^{\lambda} x\right)
$$

It follows that

$$
\sin (n x)=n^{\mathrm{i} \hat{x} \hat{p}} \sin x .
$$

Therefore, ignoring for now the question of the convergence of the sum, we deduce that

$$
\sum_{n=1}^{\infty} \frac{\sin (n x)}{n}=\sum_{n=1}^{\infty} \frac{n^{\mathrm{i} \hat{x} \hat{p}}}{n} \sin x=\zeta(1-\mathrm{i} \hat{x} \hat{p}) \sin x .
$$

Thus, the action of the Riemann dilation operator $\zeta(1-\mathrm{i} \hat{x} \hat{p})$ on a trigonometric function generates a Fourier series. [Note that we do not define an operator of the form $\zeta(z+\mathrm{i} \hat{x} \hat{p})$ as a Taylor expansion of $\zeta(s)$ about $s=z$ in powers of $\mathrm{i} \hat{x} \hat{p}$. Such an expansion may diverge.] In this example, the left side is the Fourier representation for the linear function $(\pi-x) / 2$, and from the relation $\hat{h}_{\mathrm{BK}}=2 \hat{x} \hat{p}-\mathrm{i}$ we observe that $1-\mathrm{i} \hat{x} \hat{p}=\frac{1}{2}\left(3-\mathrm{i} \hat{h}_{\mathrm{BK}}\right)$. We therefore deduce the identity (2). Hence if the operator $\zeta(1-\mathrm{i} \hat{x} \hat{p})$ were invertible, we would expect the relation

$$
\frac{1}{\zeta(1-\mathrm{i} \hat{x} \hat{p})} \frac{\pi-x}{2}=\sin x
$$

to hold. However, since $\hat{x} \hat{p}$ is the dilation generator, it cannot change the power of $x$ on the left side, so we arrive at a contradiction. This suggests that the operator $\zeta(1-\mathrm{i} \hat{x} \hat{p})$ cannot be inverted because its spectrum contains at least one zero eigenvalue.

Before we proceed to inspect the locations of the zeros, let us check the consistency of (2) without relying on the summation representation of the zeta function. For this purpose we use the integral representation

$$
\zeta(s)=\frac{\Gamma(1-s)}{2 \pi \mathrm{i}} \int_{C} \frac{t^{s-1}}{\mathrm{e}^{-t}-1} \mathrm{~d} t
$$

for the zeta function and

$$
\frac{1}{\Gamma(1-s)}=\frac{1}{2 \pi \mathrm{i}} \int_{C} \mathrm{e}^{t} t^{s-1} \mathrm{~d} t
$$

for the reciprocal of the Gamma function. Because $s$ appears in two different ways in (9) our strategy is to check the validity of

$$
\frac{1}{\Gamma(\mathrm{i} \hat{x} \hat{p})} \frac{\pi-x}{2}=\frac{\zeta(1-\mathrm{i} \hat{x} \hat{p})}{\Gamma(\mathrm{i} \hat{x} \hat{p})} \sin x
$$

to infer (2). For the left side we deduce that

$$
\frac{1}{\Gamma(\mathrm{i} \hat{x} \hat{p})} \frac{\pi-x}{2}=\frac{1}{2 \pi \mathrm{i}} \int_{C} \mathrm{e}^{t} t^{-\mathrm{i} \hat{x} \hat{p}}\left(\frac{\pi-x}{2}\right) \mathrm{d} t=\frac{1}{2 \pi \mathrm{i}} \int_{C} \mathrm{e}^{t}\left(\frac{\pi-t^{-1} x}{2}\right) \mathrm{d} t=-\frac{1}{2} x .
$$


The constant term $\pi / 2$ has been annihilated here because of the pole of $\zeta(s)$ at $s=1$. On the other hand, expanding $\sin x$ in a power series, we deduce from

$$
\frac{\zeta(1-\mathrm{i} \hat{x} \hat{p})}{\Gamma(\mathrm{i} \hat{x} \hat{p})} x^{n}=\frac{1}{2 \pi \mathrm{i}} \int_{C} \frac{t^{-\mathrm{i} \hat{x} \hat{p}}}{\mathrm{e}^{-t}-1} x^{n} \mathrm{~d} t=\frac{1}{2 \pi \mathrm{i}} \int_{C} \frac{t^{-n}}{\mathrm{e}^{-t}-1} x^{n} \mathrm{~d} t=\frac{\zeta(1-n)}{\Gamma(n)} x^{n}
$$

that

$$
\frac{\zeta(1-\mathrm{i} \hat{x} \hat{p})}{\Gamma(\mathrm{i} \hat{x} \hat{p})} \sin x=\sum_{n=1}^{\infty} \frac{\zeta(2(1-n))}{(2 n-1) ! \Gamma(2 n-1)} x^{2 n-1} .
$$

Since the right side of $(10)$ must equal $-\frac{1}{2} x$, we infer that $\zeta(0)=-\frac{1}{2}$, and that $\zeta(-2)=$ $\zeta(-4)=\cdots=0$. Conversely, from these elementary facts about the zeta function we infer the consistency of (8).

An essentially identical line of argument leads to the observation that

$$
\begin{gathered}
\zeta(2-\mathrm{i} \hat{x} \hat{p}) \cos x=\frac{\pi^{2}}{6}-\frac{\pi x}{2}+\frac{x^{2}}{4}, \\
\zeta(3-\mathrm{i} \hat{x} \hat{p}) \sin x=\frac{\pi^{2} x}{6}-\frac{\pi x^{2}}{4}+\frac{x^{3}}{12},
\end{gathered}
$$

and so on. Thus, for each of the Clausen functions in (3) and (4) we obtain a corresponding representation in the form of an operator $\zeta(N-\mathrm{i} \hat{x} \hat{p})$ acting on a trigonometric function, for $N$ a positive integer. Each of these relations reveals some information about the values of $\zeta(s)$ for real integral values of $s$.

4. As a slightly shorter way to do the analysis above, we observe that since $\mathrm{i} \hat{x} \hat{p} x^{\alpha}=\alpha x^{\alpha}$, and since $\zeta(s)$ is analytic except for a simple pole at $s=1$, we have $\zeta(N-\mathrm{i} \hat{x} \hat{p}) x^{n}=\zeta(N-n) x^{n}$. However, one must be careful about the existence of the pole. To illustrate this, we consider the example $\zeta(1-\mathrm{i} \hat{x} \hat{p}) \sin x$. Expanding the sine series, and assuming the interchangeability of the two limits, we obtain

$$
\zeta(1-\mathrm{i} \hat{x} \hat{p}) \sum_{n=1}^{\infty} \frac{x^{2 n-1}}{(2 n-1) !}=\sum_{n=1}^{\infty} \zeta(1-\mathrm{i} \hat{x} \hat{p}) \frac{x^{2 n-1}}{(2 n-1) !}=\sum_{n=1}^{\infty} \zeta(2-2 n) \frac{x^{2 n-1}}{(2 n-1) !}=-\frac{1}{2} x,
$$

which shows that term-by-term application of the differential operator $\zeta(1-\mathrm{i} \hat{x} \hat{p})$ is not permissible because we have missed the constant term $\pi / 2$ associated with the pole of $\zeta(s)$. In fact, for each of the examples discussed above, interchanging the limits leaves out just one term corresponding to the pole of $\zeta(s)$; that is, one term on the left side that is annihilated by $\Gamma(\mathrm{i} \hat{x} \hat{p}+1-N)^{-1}$. This term is the only parity-violating term; while each term on the left side of (4) has odd parity, one term on the right side has even parity. Similarly, while each term on the left side of (3) has even parity, one term on the right side has odd parity. Thus, in (11) the term $\pi x / 2$ on the right side violates parity, and similarly in (12) the term $\pi x^{2} / 4$ on the right side violates parity. Hence, the commutator of the two limits gives the parity-breaking term resulting from summing the series.

We remark that the term-by-term application of the operator respects both parity and analyticity. To illustrate this, we consider the series in (6). Observe that each term in the series on the left side has odd parity and the right side is also odd, so that there is no violation of parity. One might expect that term-by-term application of $\zeta(-\mathrm{i} \hat{x} \hat{p})$ on the 
power-series expansion of $\sin x$ is permissible. However, while each term in the series on the left side is analytic and vanishes at $x=0$, the right side diverges like $1 / x$ as $x \rightarrow 0$. Indeed,

$$
\begin{aligned}
\sum_{n=0}^{\infty} \zeta(-\mathrm{i} \hat{x} \hat{p}) \frac{(-1)^{n}}{(2 n+1) !} x^{2 n+1} & =\sum_{n=0}^{\infty} \zeta(-2 n-1) \frac{(-1)^{n}}{(2 n+1) !} x^{2 n+1} \\
& =\sum_{n=0}^{\infty}(-1)^{2 n+1} \frac{B_{2 n+2}}{2 n+2} \frac{(-1)^{n}}{(2 n+1) !} x^{2 n+1} \\
& =\frac{1}{x} \sum_{n=0}^{\infty} \frac{\mathrm{i}^{2 n+2}}{(2 n+2) !} B_{2 n+2} x^{2 n+2} \\
& =\frac{1}{x} \sum_{k=2}^{\infty} \frac{1}{k !} B_{k}(\mathrm{i} x)^{k} \\
& =\frac{1}{x}\left[\sum_{k=0}^{\infty} \frac{1}{k !} B_{k}(\mathrm{i} x)^{k}-1-\mathrm{i} B_{1} x\right] .
\end{aligned}
$$

Therefore, from the generating function $\sum_{k=0}^{\infty} B_{k} x^{k} / k !=x /\left(\mathrm{e}^{x}-1\right)$ with $B_{1}=-\frac{1}{2}$ we get

$$
\sum_{n=0}^{\infty} \zeta(-\mathrm{i} \hat{x} \hat{p}) \frac{(-1)^{n}}{(2 n+1) !} x^{2 n+1}=\frac{\sin x}{2(1-\cos x)}-\frac{1}{x} .
$$

Remarkably, we recover the right side of (1), but with its singularity removed. Moreover, the singular term in the right side of (1) corresponds to the pole of $\zeta(s)$ at $s=1$. This is the only term that is annihilated by the action of $\Gamma(1+\mathrm{i} \hat{x} \hat{p})^{-1}$.

Analogous results can be seen in other examples, for instance, in

$$
\zeta(-1-\mathrm{i} \hat{x} \hat{p}) \cos x=\sum_{n=1}^{\infty} n \cos (n x)=-\frac{1}{2(1-\cos x)} .
$$

Once again, there is no parity violation but the right side is singular at $x=0$ and behaves like $-1 / x^{2}$, while each of the summands in the middle term is well behaved. On the other hand, by interchanging the order of differentiation and summation associated with the Taylor expansion of $\cos x$ we obtain

$$
\sum_{n=0}^{\infty} \zeta(-1-\mathrm{i} \hat{x} \hat{p}) \frac{(-1)^{n}}{(2 n) !} x^{2 n}=-\sum_{n=0}^{\infty} \frac{(2 n+1)(\mathrm{i} x)^{2 n}}{(2 n+2) !} B_{2 n+2} .
$$

Then a calculation like that in (13) leads to the same conclusion that

$$
\sum_{n=0}^{\infty} \zeta(-1-\mathrm{i} \hat{x} \hat{p}) \frac{(-1)^{n}}{(2 n) !} x^{2 n}=-\frac{1}{2(1-\cos x)}+\frac{1}{x^{2}},
$$

and the singularity at the origin has been removed.

5. The analysis presented here can be extended to more general Dirichlet $L$-functions. These are functions expressible in the form

$$
L_{\chi}(s)=\sum_{n=1}^{\infty} \frac{\chi(n)}{n^{s}}
$$


for $\operatorname{Re}(s)>1$, and otherwise can be defined by their analytic continuations. Here $\chi(n)$ denotes a Dirichlet character, which is a function from integers to complex numbers satisfying the multiplicative property that $\chi(m n)=\chi(m) \chi(n)$, the periodicity that $\chi(n)=\chi(n+k)$ for some positive $k$, and the condition that if $n$ and $k$ are relative primes then $\chi(n) \neq 0$ but otherwise $\chi(n)=0$. Thus, for $k=1$ we have $\chi(n)=1$ for all $n$ and $L_{\chi}(s)$ reduces to the Riemann zeta function.

As a simple example other than the Riemann zeta function, let us consider the Dirichlet beta function arising from considering the period $k=4$. Specifically, for $\operatorname{Re}(s)>1$ the Dirichlet beta function is defined by the series

$$
\beta(s)=\sum_{n=0}^{\infty} \frac{(-1)^{n}}{(2 n+1)^{s}}
$$

from which we deduce that

$$
\beta(-\mathrm{i} \hat{x} \hat{p}) \sin x=\sum_{n=0}^{\infty}(-1)^{n}(2 n+1)^{\mathrm{i} \hat{x} \hat{p}} \sin x=\sum_{n=0}^{\infty}(-1)^{n} \sin ((2 n+1) x)=0,
$$

where the vanishing of the alternating sine series here can be deduced by using Euler summation. On the other hand, interchanging the order of summation and differentiation in the series expansion of $\sin x$ gives

$$
\beta(-\mathrm{i} \hat{x} \hat{p}) \sin x=\sum_{n=0}^{\infty} \frac{(-1)^{n}}{(2 n+1) !} \beta(-(2 n+1)) x^{2 n+1},
$$

from which we deduce that $\beta(-n)=0$ for all positive odd $n$, without explicitly relying on analytic continuation. Note that the interchange of the limits is permissible in this example because there is no pole contribution.

An analogous calculation shows that

$$
\beta(-\mathrm{i} \hat{x} \hat{p}) \cos x=\sum_{n=0}^{\infty}(-1)^{n}(2 n+1)^{\mathrm{i} \hat{x} \hat{p}} \cos x=\sum_{n=0}^{\infty}(-1)^{n} \cos ((2 n+1) x)=\frac{1}{2 \cos x},
$$

whereas by interchanging the limits we find that

$$
\beta(-\mathrm{i} \hat{x} \hat{p}) \cos x=\sum_{n=0}^{\infty} \frac{(-1)^{n}}{(2 n) !} \beta(-2 n) x^{2 n} .
$$

Comparing these two we deduce that $\beta(-n)=E_{n} / 2$ for all positive even $n$. This result can also be obtained by considering

$$
\beta(1-\mathrm{i} \hat{x} \hat{p}) \sin x=\sum_{n=0}^{\infty} \frac{(-1)^{n}}{2 n+1} \sin ((2 n+1) x)=\frac{1}{2} \mathrm{i}\left[\tan ^{-1}\left(\mathrm{e}^{-\mathrm{i} x}\right)-\tan ^{-1}\left(\mathrm{e}^{\mathrm{i} x}\right)\right],
$$

and comparing this with

$$
\beta(1-\mathrm{i} \hat{x} \hat{p}) \sin x=\sum_{n=0}^{\infty} \frac{(-1)^{n}}{(2 n+1) !} \beta(-2 n) x^{2 n+1} .
$$


6. In the foregoing analysis we have only considered one class of operator-valued zeta functions, namely, zeta functions evaluated at a linear function of the dilation operator. This class of operators is suitable in the context of Fourier analysis [7]. It appears that the action of this class of operators on trigonometric functions only yields information about $\zeta(s)$ for real $s$ although further study is required to clarify this point. In this connection, we note that the matrix elements of, for example, $\zeta(1-\mathrm{i} \hat{x} \hat{p})$, viewed as an operator acting on the Hilbert space of square-integrable functions on $[0, \pi]$, in the standard sine basis $\{\sqrt{2 / \pi} \sin (n x)\}$, is given by

$$
\zeta_{m n}= \begin{cases}n / m & \text { if } n \text { divides } m \\ 0 & \text { otherwise }\end{cases}
$$

Thus, the matrix $\left\{\zeta_{m n}\right\}$ encodes the information about factorisation of integers. This suggests that it might be possible to extract more information by studying further properties of the class of operator-valued zeta functions considered here.

Evidently, there are many other operator-valued zeta functions that one might consider. For instance, the action of $\zeta\left(\hat{p}^{2}+\hat{x}^{2}\right)$ on Hermite polynomials might yield further results on the zeta function. As another example, if we let $\hat{a}=(\hat{x}+\mathrm{i} \hat{p}) / \sqrt{2}$ denote the standard annihilation operator and $|s\rangle, s \in \mathbb{C}$, a coherent state, we then have $\zeta(\hat{a})|s\rangle=\zeta(s)|s\rangle$. Thus, if the action of the operator $\zeta(\hat{a})$ were implementable in a laboratory, then one would see the coherent light being absorbed whenever $s$ is a zero of the zeta function.

To conclude, we have shown that by studying the action of Riemann dilation operators on trigonometric functions, we are able to infer some properties of the Riemann zeta function. Of course, the properties of $\zeta(s)$ inferred here are already known. Nevertheless, we were able to determine, for example, the locations of the trivial zeros from elementary Fourier analysis without relying explicitly on the analytic continuation of the zeta function. This suggests that further research into actions of operator-valued zeta functions may yield interesting new results.

\section{Acknowledgement}

DCB thanks the Russian Science Foundation for support (project 16-11-10218). The authors thank J. Keating for suggesting the idea of examining other Dirichlet $L$-functions.

[1] Bender, C. M., Brody, D. C. \& Müller, M. P. (2017) Hamiltonian for the zeros of the Riemann zeta function. Physical Review Letters 118, 130201.

[2] Bender, C. M. \& Brody, D. C. (2018) Asymptotic analysis of a pseudo-Hermitian Riemann-zeta Hamiltonian. Journal of Physics A 51, 135203.

[3] Berry, M. V. \& Keating, J. P. (1999) $H=x p$ and the Riemann zeros. In Supersymmetry and Trace Formulae: Chaos and Disorder. Edited by I. V. Lerner et al. (New York: Kluwer Academic / Plenum).

[4] Abramowitz, M. \& Stegun, I. A. (1983) Handbook of Mathematical Functions with Formulas, Graphs, and Mathematical Tables. 23.1.17 and 23.1.18 (New York: Dover). p. 1005.

[5] Clausen, T. (1832) Über die Function $\sin \phi+\left(1 / 2^{2}\right) \sin 2 \phi+\left(1 / 3^{2}\right) \sin 3 \phi+$ etc. Journal für die reine und angewandte Mathematik $\mathbf{8}, 298-300$.

[6] Apostol, T. M. (1951) On the Lerch zeta function. Pacific Journal of Mathematics 1, 161-167. 
[7] Brody, D. C. (2018) Biorthogonal systems on unit interval and zeta dilation operators. Journal of Physics A 51, 285202. 